\title{
RESEARCH
}

Open Access

\section{Tendon regeneration and muscle hypotrophy after isolated Gracilis tendon harvesting - a pilot study}

Anne Flies ${ }^{1}$, Timm Denecke ${ }^{2}$, Natascha Kraus ${ }^{3}$, Philipp Kruppa ${ }^{4}$, Matthew T. Provencher ${ }^{5}$, Roland Becker ${ }^{6}$ and Sebastian Kopf $^{*}$

\begin{abstract}
Purpose: The gracilis tendon (GT) is a commonly used autologous graft in Orthopaedic surgery. The majority of information on knee function and outcomes after hamstring harvest is related to both semitendinosus and GT harvest. Little is known regarding isolated harvest of a GT. It was hypothesized that isolated GT harvest would lead to altered gait patterns (e.g. augmented anterior-posterior translation or rotation in the tibiofemoral joint) and consequently a higher prevalence of cartilage lesions and meniscal tears in knees.

Methods: GT harvesting was performed on patients with chronic acromioclavicular joint instability without previous knee injuries or surgeries. MRI of both knees and thighs were performed. Knee MRI were evaluated using the Whole-Organ Magnetic Resonance Imaging Score (WORMS). Inter- and intraobserver reliabilities, cross-sectional areas of different muscles, fatty infiltration of the gracilis muscle (GM) and GT regeneration were evaluated. The contralateral limb served as reference. The observers were blinded towards the identity of the patients and the operatively treated side.

Results: After a mean time of 44 months after surgery testing was performed on 12 patients. No significant side-toside differences were found using WORMS, although there was a trend towards increased cartilage lesions after GT harvest (median healthy knee 4.8 and GT harvested knee $7.8 p=0.086$ ). Inter- and intraobserver repeatability was high with 0.899 (95\% confidence interval (Cl) 0.708-0.960) and 0.988 (95\% Cl 0.973-0.995), respectively. A significant hypotrophy of the GM with a mean decrease of $25.3 \%, 18.4 \%$ and $16.9 \%$ occurred at $25 \%$ ( $p=0.016$ ), 50\% ( $p=$ $0.007)$ and $75 \%(p=0.002)$ of the length of the femur from distal. No compensatory hypertrophy of other thigh muscles or increased fatty infiltration of the GM was found. Tendon regeneration took place in eight out of 12 patients. In case of regeneration, the regenerated tendon inserted in a more proximal place.

Conclusion: Isolated harvest of the GT for shoulder procedures did not affect knee MRI significantly indicating therefore in general suitable graft utilization for surgeries outside of the knee. GT regenerated in most patients with just a more proximal insertion and a hypotrophy of the muscle belly.
\end{abstract}

Keywords: Gracilis, Autograft, Tendon harvest, MRI, Radiologic outcome

\footnotetext{
*Correspondence: s.kopf@klinikum-brandenburg.de

Research performed at Charité - University Medicine Berlin

${ }^{6}$ Center of Orthopaedics and Traumatology, Brandenburg Medical School

Theodor Fontane, Hospital Brandenburg an der Havel, 14770 Brandenburg

an der Havel, Germany

Full list of author information is available at the end of the article
}

\section{Springer Open}

(c) The Author(s). 2020 Open Access This article is licensed under a Creative Commons Attribution 4.0 International License, which permits use, sharing, adaptation, distribution and reproduction in any medium or format, as long as you give appropriate credit to the original author(s) and the source, provide a link to the Creative Commons licence, and indicate if changes were made. The images or other third party material in this article are included in the article's Creative Commons licence, unless indicated otherwise in a credit line to the material. If material is not included in the article's Creative Commons licence and your intended use is not permitted by statutory regulation or exceeds the permitted use, you will need to obtain permission directly from the copyright holder. To view a copy of this licence, visit http://creativecommons.org/licenses/by/4.0/. 


\section{Background}

The gracilis tendon (GT) is a popular graft source for reconstructive procedures in orthopedic surgery $[5,17,19,29]$. To our knowledge, the consequences of isolated GT harvesting in healthy knees remains unclear. Patients were only examined with torn ACL and reconstruction with isolated ST or combined ST and GT graft. Consequently, the outcome parameters were influenced by several factors: injury to the knee with damage of further structures (menisci, collateral ligaments), additional harvest of the ST tendon, surgery (ACL reconstruction), and rehabilitation of the knee. In studies on patients after rupture and reconstruction of the ACL with ST or ST and GT graft a substantially higher risk for developing osteoarthritis (OA) of the knee joint was shown $[7,18,36]$. Although it remains unclear whether development of OA was primarily because of the persisting rotational instability after ACL reconstruction [36] or also because of insufficient rotational control after harvest of hamstrings autograft [7]. The regenerative potential of ST and GT after harvesting for ACL reconstruction was for the first time reported by Cross et al. in 1992 [6]. Strength deficits were found after harvesting of the GT and ST tendon and as a consequence a compensatory hypertrophy of the hamstrings muscles [3, 10, 16]. But, it remains unclear whether deficits in thigh muscle strength were created by isolated harvesting of the GT. Only mild fatty infiltration was described after hamstrings tendon harvesting. It was hypothesized that further fatty infiltration did not take place because the functionality of the muscles was at least partially preserved [32, 37]. To sum up, the consequences of hamstrings tendon harvesting on the ipsilateral knee joint were analyzed by several studies. However, all of them presented a large number of factors influencing the outcome. Thus, the aim of the present study was to analyze the radiologic consequences of isolated GT harvest of otherwise healthy, uninjured knees.

As a primary outcome it was hypothesized that altered kinematics after GT harvesting would lead to a higher prevalence of cartilage lesions and meniscal tears in knees after GT harvest and therefore show a higher WORMS. As secondary outcomes it was hypothesized that after harvesting the GT, a tendon-like structure would regenerate, but lead to a hypotrophy and fatty infiltration of the gracilis muscle (GM). In addition, a compensatory hypertrophy of the other hamstrings muscles was hypothesized.

\section{Methods}

In this retrospective cohort pilot study patients with chronic ACJ instability who underwent stabilization with autologous GT graft, in our clinic between 2007 and 2014, were considered for testing. The study was approved by the ethics committee of our institution (no. EA2/104/12). The study was registered at the German clinical trial register (no. DRKS00007100).
Written consent was given by all patients who met the inclusion criteria. Criteria for inclusion were: 1) stabilization of their ACJ with isolated GT at least 1 year before follow-up, 2) aged between 18 and 60 years, 3) no history of previous surgeries or injuries on both knees, and 4) ability to perform the MRI analysis (e.g. no claustrophobia).

Apart from the usual demographic data the Marx Activity Rating Scale was collected to describe our study population [20].

\section{Surgical technique}

GT was harvested under general anesthesia and a single shot perioperative antibiotic. The tendon harvesting was performed by different well-experienced knee and shoulder surgeons. The patients were placed in the beach chair position because of the following intervention on the ACJ. After full relaxation a tourniquet was applied to the thigh. An approximately $2,5 \mathrm{~cm}$ longitudinal incision was made over the pes anserine. Then the sartorius aponeurosis was identified and an incision was made proximal to the GT over the length of approximately $4 \mathrm{~cm}$. The GT was identified and released at its musculotendinous junction with an open tendon stripper. Finally the distal attachment was dissected and released from the bone [29].

\section{MRI examination}

MRI of both knees and thighs were performed in dedicated multi-channel knee and surface coils using a 1.5 Tesla system (Avanto, Siemens, Erlangen, Germany). Sequences allowing a good evaluation of the cartilage, menisci and ligaments were chosen for the knee and of the tendons and muscles for the thighs (Supplement 1). MR images were evaluated using the Osirix software (Pixmeo, Bernex, Switzerland).

First, it was checked if the GT was harvested and not accidentally the ST. Knee MRIs were evaluated separately by three different researchers allowing the calculation of the interobserver repeatability. A second evaluation was performed 6 weeks later by the third researcher in order to calculate the intraobserver repeatability. The observers were blinded with regard to the identity of the patients and the operatively treated side. For the evaluation the Whole-Organ Magnetic Resonance Imaging Score (WORMS) was used [26]. Fourteen features of the knee joint were scored independently. Five of these features (cartilage, subarticular bone marrow abnormality, subarticular cysts, subarticular bone attrition, marginal osteophytes) were evaluated in 15 different regions (Table 1). Consequently, a total combined score as well as scores for the different features and regions were calculated. A healthy knee joint was scored 0 . The higher the score the more abnormalities presented the knee joint. The worst possible score was 332. The contralateral healthy knee of 
Table 1 WORMS features and regional subdivision of the knee joint. Features marked with * were evaluated in the different regions. Cartilage, bone attrition and osteophytes were not evaluated in the S-region

\begin{tabular}{|c|c|c|}
\hline Features & Regional Subdivision & \\
\hline articular cartilage integrity* & Patellofemoral joint (PFJ) & medial patella (MP) \\
\hline subarticular bone marrow abnormality* & & lateral patella (LP) \\
\hline subarticular cysts* & & anterior medial femoral condyle (MFa) \\
\hline subarticular bone attrition* & & anterior lateral femoral condyle (LFa) \\
\hline marginal osteophytes* & Medial tibiofemoral joint (MTFJ) & posterior femoral condyle (MFp) \\
\hline medial meniscal integrity & & central medial femoral condyle (MFc) \\
\hline lateral meniscal integrity & & anterior medial tibial plateau (MTa \\
\hline anterior cruciate ligament integrity & & central medial tibial plateau (MTc) \\
\hline posterior cruciate ligament integrity & & posterior medial tibial plateau (MTp) \\
\hline medial collateral ligament integrity & Lateral tibiofemoral joint (LTFJ) & central lateral femoral condyle (LFc) \\
\hline lateral collateral ligament integrity & & posterior lateral femoral condyle (LFp) \\
\hline synovitis/effusion & & anterior lateral tibial plateau (LTa) \\
\hline intraarticular loose bodies & & central lateral tibial plateau (LTc) \\
\hline periarticular cysts/bursitis & & posterior lateral tibial plateau (LTp) \\
\hline & Subspinous region & $\begin{array}{l}\text { portion of the tibial plateau beneath } \\
\text { the tibial spines (S-region) }\end{array}$ \\
\hline
\end{tabular}

each patient served as reference for the gracilis tendon harvested knee. Furthermore, a correlation analysis between the WORMS of the knee and time of follow-up was performed. In the T1-weighted axial images of the thigh, the cross-sectional area (CSA) of different muscles was measured at four different heights; the distal femoral growth plate, $25 \%, 50 \%$ and $75 \%$ of the length of the femur from distal. The CSA was measured using the closed polygon tool of OSIRIX (Fig. 1a and b, Table 2). In addition to CSA of the muscles, the fatty infiltration of the GM was evaluated as described by Engelken et al. [9]. These measurements were conducted using the ImageJ software [30]. Therefore, a slice at the transition from the middle to the distal third of the length of the femur in an axial t1-weighted sequence was chosen. First, reference measurements of pure fat and pure muscle tissue were

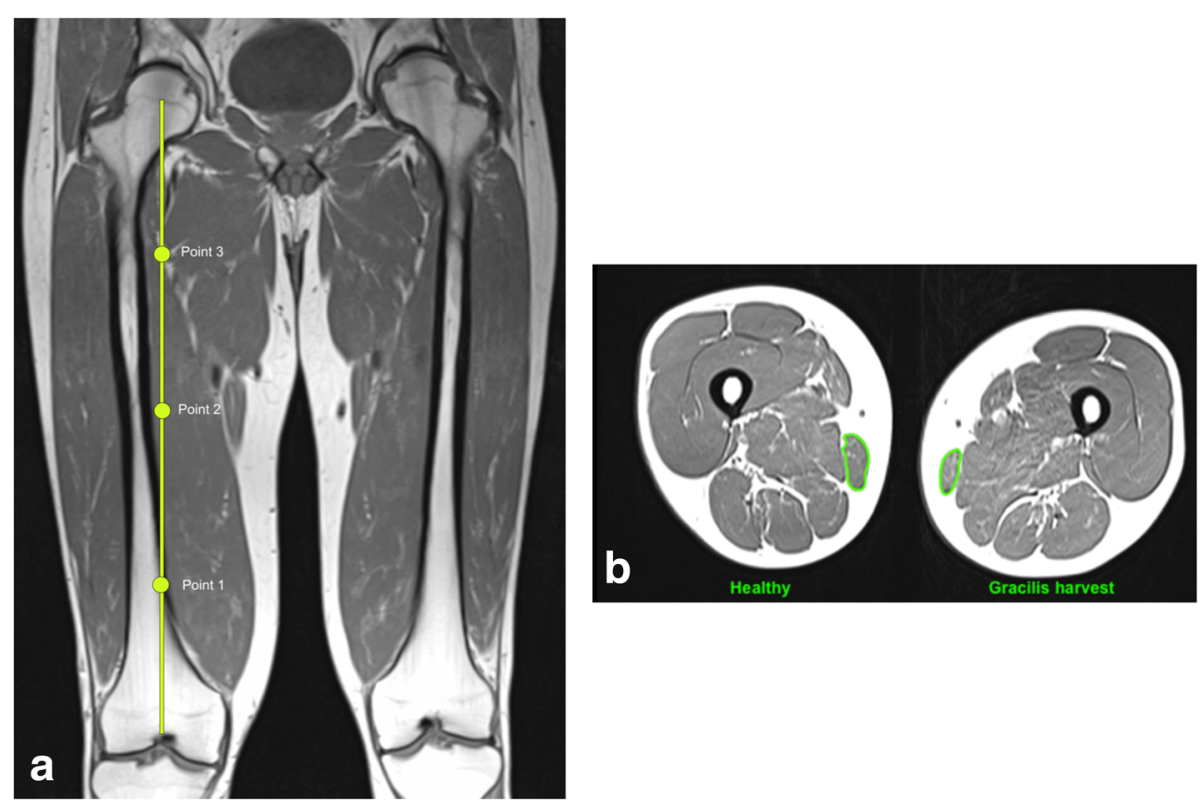

Fig. $1 \mathbf{a}$ and $\mathbf{b}$ Measurement of the CSA of the gracilis muscle. The bullets indicate $25 \%, 50 \%$ and $75 \%$ of the length of the femur from distal. Gracilis harvest $=$ limb with previous harvest of the gracilis tendon, Healthy = contralateral limb 
Table 2 Heights at which the cross-sectional areas of the muscles were measured from distal to proximal thigh. Empty fields indicate that the muscle was not identifiable

\begin{tabular}{llllllll}
\hline Height & \multicolumn{3}{l}{ Muscles } & & & & \\
\cline { 2 - 7 } & G & ST & SM & BF & Q & RF & VLNMNI \\
\hline growth plate & & & $\times$ & $\times$ & & & \\
$25 \%$ & $\times$ & $\times$ & $\times$ & $\times$ & & & \\
$50 \%$ & $\times$ & $\times$ & $\times$ & $\times$ & $\times$ & $\times$ & $\times$ \\
$75 \%$ & $\times$ & $\times$ & & & $\times$ & $\times$ & $\times$ \\
\hline
\end{tabular}

$G$ M. gracilis, ST M. semitendinosus, SM M. semimembranosus, $B F$ M. biceps femoris, $Q M$. quadriceps, $R F M$. rectus femoris, VLNM/VI Sum of the Mm. vastus medialis, vastus lateralis and vastus intermedius

performed in an oval region of interest (ROI) with approximately 200 counts. The intensity ranges in which all pixels represent pure fat or pure muscle were determined as the means \pm 2 standard deviations (SD). Next, the GM was defined as ROI with the freehand selection tool and intensity profiles were created for each pixel in this ROI. Then the pixels in the fat intensity and those in the muscle intensity range were added and a ratio (fat/muscle) was calculated. As reference, the same measurements were conducted at the contralateral thigh.

In addition, the observer checked whether the GT regenerated. It was distinguished between "no regeneration", "tendon-like regeneration", and "muscle-like regeneration "at the joint line, height of the distal femoral growth plate and at $25 \%$ of the length of the femur from distal.

\section{Statistical analyses}

This was a pilot study, which is why an a priori power analysis was not performed but it was attempted to included all available patients operated at our institution. To test the Gaussian distribution of the population, the D'Agostino \& Pearson omnibus normality test was used. To analyze for side-to-side differences the dependent $t$ test was used for parametric data and the Wilcoxon signed-rank test for non-parametric data. Parametric data are presented as mean and SD or 95\% confidence interval $(\mathrm{CI})$, whereas non-parametric data are presented as median and interquartile range (IQR). If applicable, the range was added e.g. for follow-up time and age. For correlation analyses the Pearson correlation coefficient was used for parametric data and otherwise the Spearman correlation coefficient. Predictive values were calculated as an approximation using $\mathrm{r}^{2}$.

The level of significance was $5 \%(p<0.05)$ Intra- and interclass correlations were calculated using the Intraclass Correlation Coefficient (ICC) for absolute agreement [27]. All statistical analyses were performed using GraphPad Prism Version 6.01 (GraphPad Software, Inc., San Diego, CA, U.S.A.).

\section{Results}

Sixteen patients were evaluated (14 male, 2 female). Retrospectively four patients had to be excluded because the MRIs showed accidental harvest of the ST tendon instead of the GT (Fig. 2). Demographic data is shown in Table 3. The gracilis tendon was harvested in equal parts from the right and left limb (six each).

The total combined WORMS showed a median of 6.3 points (IQR 2.5 to 10 ) for the healthy limb and 10 points (IQR 5.4 to 15.5) for the limb with GT harvest $(p=$ 0.095). The evaluation of the articular cartilage integrity of the knee joint showed a median of 4.8 (IQR 1.4 to 7.4) for healthy limbs and 7.8 (IQR 4.3 to 10.8) for limbs with GT harvest reaching almost statistical significance $(p=0.086)$. For all other features, statistical differences were higher (Table 4). Evaluation of the WORMS of the regions patellofemoral joint (PFJ), medial tibiofemoral joint (MTFJ) and lateral tibiofemoral joint (LTFJ) showed a median of 2.3 (IQR 0 to 3.8), 3.0 (IQR 0 to 3.9) and 0 (IQR 0) for the healthy limb and 3.3 (IQR 0.3 to 7.3), 3.5 (IQR 0.8 to 6.0) and 0 (IQR 0) for the GT harvested limb with a level of significance of $p=0.438$, $p=0.234$ and $p>0.05$ respectively.

Articular cartilage integrity in the PFJ, MTFJ and LTFJ showed a median of 1.5 (IQR 0 to 3.6), 3.0 (IQR 0 to 3.8 ) and 0 (IQR 0$)$ for the healthy limb and 3.3 (IQR 0 to 4.8 ), 3.5 (IQR 0.8 to 6.0 ) and 0 (IQR 0 ) for the limb with GT harvest with a level of significance of $p=0.5$, $p=0.141$ and $p>0.05$ respectively. For all other features and regions, no differences between the mean or median of the healthy and GT harvested limb were found.

Inter- and intraobserver repeatability was high with 0.899 (95\% CI 0.708 to 0.96 ) and 0.988 (95\% CI 0.973 to 0.995), respectively. A correlation between the total combined WORMS and time of follow-up could not be established $(p>0.05)$.

A tendon-like regeneration of the GT at the joint line was observed for five patients. At the height of the femoral growth plate, eight patients showed a tendon-like regeneration of the harvested GT. At 25\% of the length of the femur from distal, a muscle-like regeneration was observed for four, a tendon-like regeneration for seven and no regeneration for one patient.

The CSA of the different muscles showed a statistically significant hypotrophy of the GM at all heights apart from the growth plate where the CSA on the operated side was not measurable because of the more proximal insertion of the tendon (Table 5, Fig. 3). The mean decrease of the CSA of the GM was $25.3 \%, 18.4 \%$ and $16.9 \%$ at $25 \%, 50 \%$ and $75 \%$ of the length of the femur from distal compared to the contralateral limb. A compensatory hypertrophy of the other thigh muscles was not observed, neither for all the patients nor in the subgroup where no regeneration of the tendon took place. 


\section{Enrollment}

Assessed for eligibility
(patients after stabilization of acromioclavicular
joint between 2007 to March 2014)
$(n=34)$

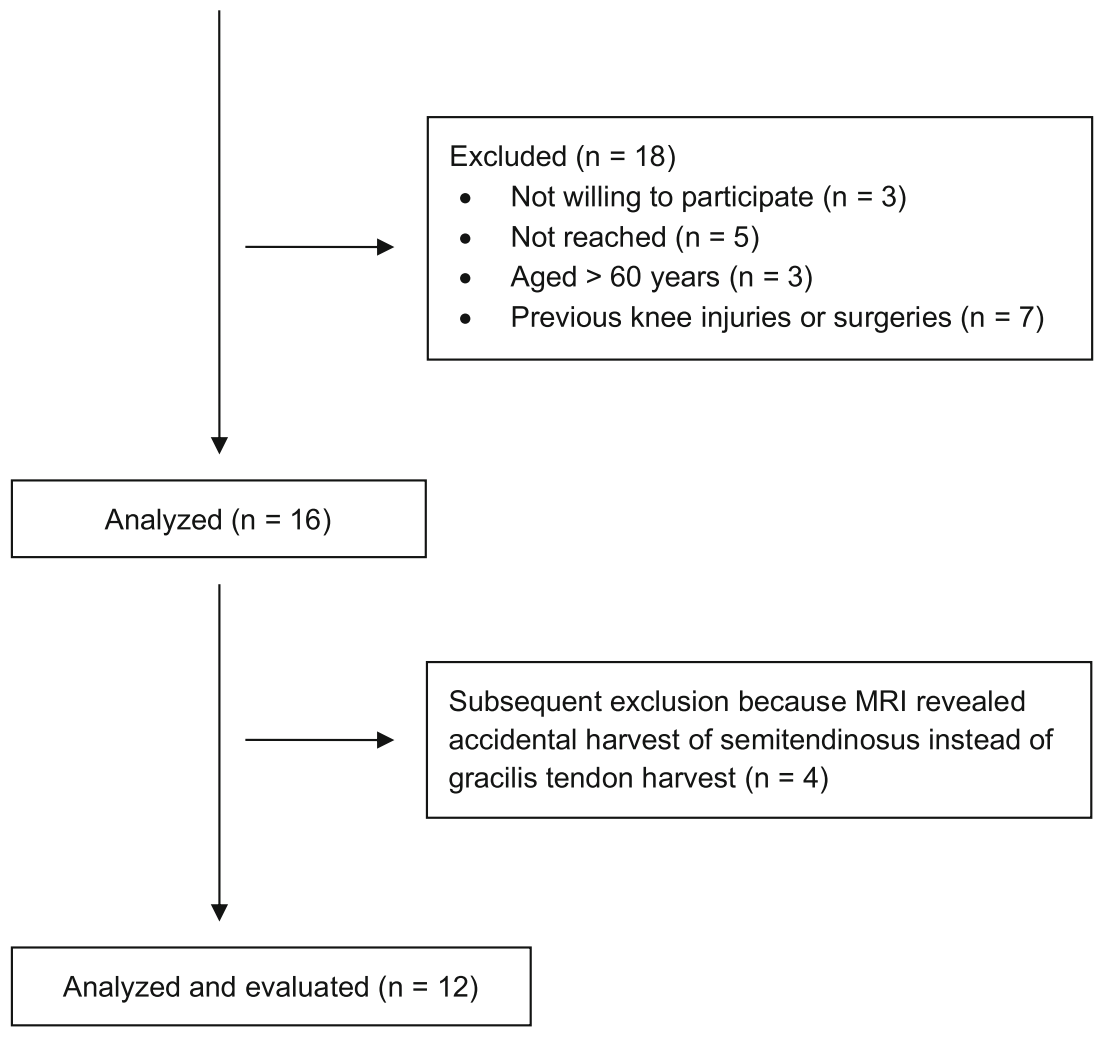

Fig. 2 Flow chart illustrating patient enrolment

An increased fatty infiltration of the GM after harvesting of its tendon was not observed. The fatty infiltration of healthy GM was 0.006 (IQR 0.001 to 0.01 ), whereas it was 0.015 (IQR 0 to 0.028$)(p>0.05)$ for the ones with the harvested tendon.

\section{Discussion}

The main finding of this study was that no statistical differences in the WORMS between the GT harvested thighs and the contralateral thighs were found. A trend towards increased cartilage lesions in the GT harvested thigh was shown in the WORMS. These lesions occurred especially in the PFJ and the MTFJ but without reaching statistical significance. The MTFJ and the lateral part of the PFJ represent also the regions in healthy knee joints with the biggest changes in cartilage thickness during loading and have therefore, irrespective of GT harvesting, a higher risk for developing OA [8]. Furthermore, changes in knee joint kinematics may be caused by harvesting of the GT and lead to early OA (e.g. smaller peak knee flexion angle, greater tibial rotation excursion, lower peak knee flexion moments) [7]. However, smaller knee flexion angles during stance phase of gait were also observed in uninjured patients

Table 3 Demographic data

\begin{tabular}{llll}
\hline & Mean & Standard Deviation & Range \\
\hline Age $[$ years] & 43 & 13 & $20-56$ \\
Follow-up Time $[\mathrm{months}]$ & 44 & 25 & $20-93$ \\
Body Mass Index $\left[\mathrm{kg} / \mathrm{m}^{2}\right]$ & 26.5 & 4.5 & $19.9-33.6$ \\
Marx Activity Rating Scale [points] & 4 & 5 & $0-13$ \\
\hline
\end{tabular}


Table 4 Results of WORMS of the knee joint of healthy and gracilis harvested limbs

\begin{tabular}{|c|c|c|c|c|c|}
\hline & $\begin{array}{l}\text { Mean/Median } \\
\text { Healthy }\end{array}$ & $\begin{array}{l}95 \%-\mathrm{Cl} / \mathrm{IQR} \\
\text { Healthy }\end{array}$ & $\begin{array}{l}\text { Mean/median Gracilis } \\
\text { Harvest }\end{array}$ & $\begin{array}{l}95 \%-C I / I Q R \text { Gracilis } \\
\text { Harvest }\end{array}$ & $\boldsymbol{p}$-value \\
\hline Total combined score & 6.3 & $2.5-10$ & 10 & $5.4-15.5$ & 0.095 \\
\hline Cartilage & 4.8 & $1.4-7.4$ & $4.3-10.8$ & $4.3-10.8$ & 0.086 \\
\hline Bone marrow abnormality & 0 & $0-0$ & 0 & $0-0$ & 0.375 \\
\hline Subarticular cysts & 0 & $0-0$ & 0 & $0-0$ & $\bullet$ \\
\hline Subarticular bone attrition & 0 & $0-0$ & 0 & $0-0$ & $\bullet$ \\
\hline Osteophytes & 0 & $0-0$ & 0 & $0-0$ & $\overrightarrow{0} .999$ \\
\hline Menisci & 0 & $0-0$ & 0 & $0-2.5$ & 0.125 \\
\hline $\begin{array}{l}\text { Ligaments (cruciates and } \\
\text { collaterals) }\end{array}$ & 0 & $0-0$ & 0 & $0-0$ & $\bullet$ \\
\hline Synovitis/effusion $^{a}$ & 0.3 & $-0.1-0.5$ & 0.3 & $-0.1-0.5$ & $\overrightarrow{>} .999$ \\
\hline Loose bodies & 0 & $0-0$ & 0 & $0-0$ & $\bullet$ \\
\hline Periarticular cysts / bursitis & 0.5 & $0.0-1.0$ & 0.5 & $0.0-1.0$ & $>0.999$ \\
\hline
\end{tabular}

no difference between the two limbs, cannot calculate a paired t-test or Wilcoxon test. ${ }^{\mathrm{a} G a u s s i a n}$ distribution. $\mathrm{Cl}$ confidence interval, IQR Interquartile range

with early patellofemoral OA because the surface through which patellofemoral loads are distributed is limited [7, 12]. Greater tibial rotation excursion after ACL reconstruction is potentially due to insufficient rotational control after hamstrings autograft and might be part of the mechanism after GT harvest too [7]. The small number of patients and the short time of followup might be an explanation for the missing statistical significance in the present study. However, a correlation between the total combined WORMS and time of follow-up was not found in the present study either. Thus, it remains unclear whether the tendency towards early cartilage lesions is a coincidence or due to tendon harvest.

High inter- and intraobserver repeatability in this study show good applicability of the WORMS in clinical use and the quality of the assessment.

Measurements of the cross-sectional area of the GM showed hypotrophy irrespective of regeneration of its tendon. However, no hypertrophy of the other thigh muscles, and especially the other hamstrings muscles, was seen. Hypotrophy of the GM in patients with GT and ST tendon harvest for ACL reconstruction was confirmed in the past $[2,15,16,28,39]$. On the other side, no decrease of the CSA of the G and ST muscle after harvesting for ACL reconstruction was found $10 \mathrm{~cm}$ above the joint, but this is distal to the main portion of the muscle bellies of most patients [31]. The findings of the present study contradict with prior published data showing a hypertrophy of the BF and SM muscles if no regeneration of the GT had occurred [3, 10, 14]. Hypertrophy of the SM muscle and long head of the BF muscle was even found irrespective of ST tendon and GT regeneration [16]. However, most of them harvested both, the ST and GT, tendons of muscles that have similar functions and therefore might lead to a higher deficit in muscle strength and a compensatory hypertrophy of the other hamstrings muscles. Findings of Eriksson et al. contradict this theory. After isolated harvesting of the ST patients without tendon regeneration showed hypertrophy of the SM muscle [10]. Factors that may implicate a compensatory hypertrophy of the SM and BF muscles are harvesting of multiple tendons (ST and GT) and if no regeneration of the harvested tendons takes place.

In the present study a fatty infiltration of the GM using MRI was not observed. Mild fatty infiltration (Goutallier Grade 1 or 2) of both ST and GM was shown in previous studies after harvesting both tendons in patients with ACL reconstruction [32, 37]. Histological

Table 5 Cross-sectional area of gracilis muscle

\begin{tabular}{llllll}
\hline & Mean/Median Healthy $\left(\mathrm{cm}^{\mathbf{2}}\right)$ & $95 \%-\mathrm{Cl} /$ IQR Healthy & Mean/Median Gracilis Harvest $\left(\mathrm{cm}^{\mathbf{2}}\right)$ & $95 \%$-Cl/IQR Gracilis Harvest & $\boldsymbol{p}$-value \\
\hline $\mathbf{2 5 \%}$ & 1.87 & $1.50-2.33$ & 0.96 & $0.15-1.71$ & $0.016^{*}$ \\
$\mathbf{5 0 \%}{ }^{\mathrm{a}}$ & 4.54 & $3.76-5.31$ & 3.70 & $2.68-3.76$ & $0.007^{*}$ \\
$\mathbf{7 5 \%}$ & 3.71 & $2.69-4.33$ & 2.82 & $2.18-3.54$ & $0.002^{*}$ \\
\hline
\end{tabular}

*reached statistical significance. ${ }^{a}$ Gaussian distribution. $\mathrm{Cl}$ confidence interval, IQR Interquartile range 


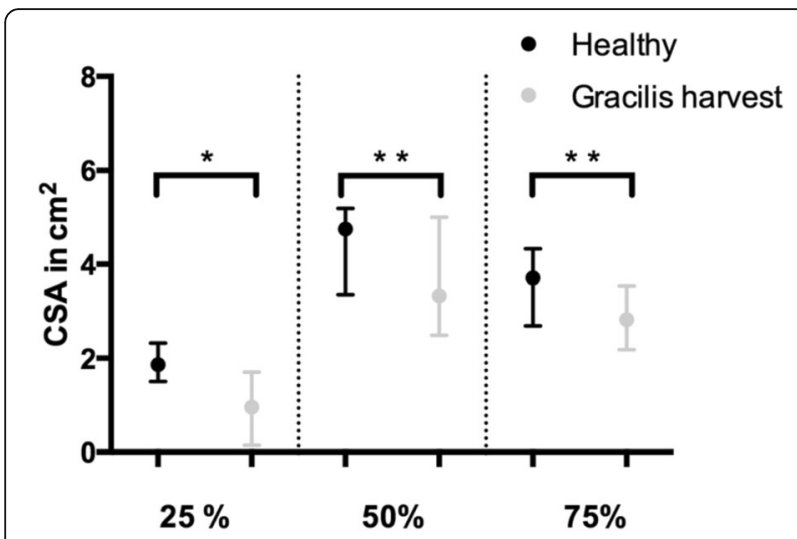

Fig. 3 Comparison of the CSA of the gracilis muscle between limbs with gracilis tendon harvest (Gracilis harvest) and contralateral limbs (Healthy) at 25\%,50\% and $75 \%$ of the length of the femur from distal. Results presented as median with IQR. ${ }^{*} p<0.05$; * $p<0.005$

analysis of the ST muscle of rabbits three, 6 and 12 months after harvesting their tendon ipsilateral showed no significant differences in fatty infiltration between both thighs (contralateral thigh was control). They hypothesized that low fatty infiltration was a sign that the muscle remained functional after tendon harvest [38].

Regeneration of the tendon of the GM took place in eight out of 12 patients. For these eight patients a tendon was detected at the distal femoral growth plate, but for only five of them a tendon was observed at the knee joint line, which lets us deduce that the regenerated tendons do not insert in their original localization, the pes anserinus superficialis, but in a more proximal position. This is in accordance with the findings of the majority of the previous studies after single ST tendon harvest or combined harvest of ST and GT. A more proximal insertion was shown in MRI [6, 28, 31, 34, 39], sonography [25] and surgical exploration [13]. Few authors, however, found in MRI or macroscopically during surgical exploration regenerated tendons at its normal insertion [1,24]. Even a tibial insertion distal of the original point of insertion of the harvested tendon was detected, but with a more proximal musculotendinous junction [4]. Latter was confirmed by several authors [15, 21, 37]. It was hypothesized that the more proximal tibial insertion and musculotendinous junction had functional consequences and would lead to strength deficits especially for deep knee flexion angles $\left(\geq 70^{\circ}\right)[1,4,22,23,33,35]$. It was hypothesized that the more proximal tibial insertion and retraction of the muscle belly would lead to a shorter knee flexion moment arm and that therefore the concerned muscles are not able to produce the same amount of force [34, 35, 39]. At lower grades of knee flexion, the BF and SM muscles are the main producers of knee flexion strength and able to compensate here a loss of ST (and G) muscle strength [6, 39]. After ACL reconstruction with ST and GT autograft a correlation between the number of regenerated tendons and the strength deficit was shown [4]. Microscopically a fibrous structure appeared after 6 months, which evolved to a structure similar to the preharvest tendon after 2 years [11, 13], but some small areas with scar tissue persisted [13]. To summarize, scientific knowledge remains controversial if it comes to tendon regeneration and functionality of the regenerated tendons.

\section{Limitations}

The present study has some limitations. First of all, more included patients would be favorable. Nevertheless, this was a pilot study looking for trends, where GT harvest might influence knee degeneration and muscle morphology of the thigh. All patients since starting with use of GT for chronic ACJ stabilization with a minimum follow-up of 1 year were tried to be included. Obviously, the risk of a type-II-statistical error was well-known. Unfortunately, the initial cohort was further decimated because of accidental harvest of the ST tendon.

A second limitation is the cross-sectional study design. Comparisons were only made between the surgical and contralateral limb. No presurgical comparisons existed and we had no data about the evolution of cartilage lesions, CSA of the thigh muscles and tendon regeneration over time. The present study cohort included only two female patients and therefore separate analyses on gender differences could not be performed. Some patients also presented short times of follow-up, which could make it difficult to detect degenerative changes or tendon regeneration. A correlation between the total combined WORMS and time of follow-up, however, could not be established. However, analysis of the radiologic consequences of isolated GT harvest in uninjured knee joints represent a novel and valuable addition to the literature.

\section{Conclusions}

Isolated harvest of the GT for shoulder procedures did not affect knee MRI significantly indicating therefore in general suitable graft utilization for surgeries outside of the knee. GT regenerated in most patients with just a more proximal insertion and a hypotrophy of the muscle belly.

\section{Supplementary information}

Supplementary information accompanies this paper at https://doi.org/10. 1186/s40634-020-00236-8.

Additional file 1: Supplement 1. Sequences of the MRI of both knees and thighs

\section{Abbreviations}

GT: Gracilis tendon; WORMS: Whole-Organ Magnetic Resonance Imaging Score; GM: Gracilis muscle; Cl: Confidence interval; ACL: Anterior cruciate 
ligament; MPFL: Medial patellofemoral ligament; ACJ: Acromioclavicular joint; OA: Osteoarthritis; PFJ: Patellofemoral joint; MP: Medial patella; LP: Lateral patella; MTFJ: Medial tibiofemoral joint; MFa: Anterior medial femoral condyle; MFc: Central medial femoral condyle; MFp: Posterior medial femoral condyle; MTa: Anterior medial tibia plateau; MTc: Central medial tibia plateau; MTp: Posterior medial tibia plateau; LTFJ: Lateral tibiofemoral joint;

LFa: Anterior lateral femoral condyle; LFc: Central lateral femoral condyle; LFp: Posterior lateral femoral condyle; LTa: Anterior lateral tibia plateau; LTc: Central lateral tibia plateau; LTp: Posterior lateral tibia plateau; CSA: Cross-sectional area; G: Gracilis; ST: Semitendinosus;

SM: Semimembranosus; BF: Biceps femoris; Q: Quadriceps; RF: Rectus femoris; VL: Vastus lateralis; VM: Vastus medialis; VI: Vastus intermedius; ROI: Region of interest; SD: Standard deviation; ICC: Intraclass Correlation Coefficient; BMl: Body mass index

\section{Acknowledgements}

$$
\text { Not applicable. }
$$

\section{Authors' contributions}

Author AF performed the examinations of the study. SK, TD and AF analyzed the data and performed the statistical analysis. NK helped with the patient recruitment. All authors substantially revised the work. The authors read and approved the final manuscript.

\section{Funding}

This work was supported by the AGA - Society for Arthroscopy and Joint Surgery under research grant No. 63.

\section{Availability of data and materials}

The datasets used and analyzed during the current study are available from the corresponding author on reasonable request.

\section{Ethics approval and consent to participate}

All procedures performed in this study were in accordance with the ethical standards of the institutional research committee and with the 1964 Helsinki declaration and its later amendments or comparable ethical standards. The study was approved by the ethics committee of our institution (no. EA2/104/ 12) and all patients gave written consent. The study was registered at the German clinical trial register (no. DRKS00007100).

Informed consent was obtained from all individual participants included in the study. Additional informed consent was obtained from all individual participants for whom identifying information is included in this article.

\section{Competing interests}

Author M. T. Provencher received IP royalties and speaker fees from Arthrex Inc. as well as consulting fees from Slack Inc. and Joint Research Foundation. All other authors declare that they have no conflict of interest.

\section{Author details}

${ }^{1}$ Center for Musculoskeletal Surgery, Charité - University Medicine Berlin, Berlin, Germany. ${ }^{2}$ Department of Radiology, Charité - University Medicine Berlin, Berlin, Germany. ${ }^{3}$ Clinic and Outpatient Clinic for Orthopaedics and Orthopaedic Surgery, University Medicine, Greifswald, Germany. ${ }^{4}$ Department of Plastic, Aesthetic and Reconstructive Microsurgery/Hand surgery, Hospital Ernst von Bergmann, Potsdam, Germany. ${ }^{5}$ The Steadman Clinic, Vail, CO, USA. ${ }^{6}$ Center of Orthopaedics and Traumatology, Brandenburg Medical School Theodor Fontane, Hospital Brandenburg an der Havel, 14770 Brandenburg an der Havel, Germany.

\section{Received: 8 January 2020 Accepted: 30 March 2020}

\section{Published online: 07 April 2020}

\section{References}

1. Ahlen M, Liden M, Bovaller A, Sernert N, Kartus J (2012) Bilateral magnetic resonance imaging and functional assessment of the semitendinosus and gracilis tendons a minimum of 6 years after ipsilateral harvest for anterior cruciate ligament reconstruction. Am J Sports Med 40:1735-1741

2. Burks RT, Crim J, Fink BP, Boylan DN, Greis PE (2005) The effects of semitendinosus and gracilis harvest in anterior cruciate ligament reconstruction. Arthroscopy 21:1177-1185
3. Carofino B, Fulkerson J (2005) Medial hamstring tendon regeneration following harvest for anterior cruciate ligament reconstruction: fact, myth, and clinical implication. Arthroscopy 21:1257-1265

4. Choi JY, Ha JK, Kim YW, Shim JC, Yang SJ, Kim JG (2012) Relationships among tendon regeneration on MRI, flexor strength, and functional performance after anterior cruciate ligament reconstruction with hamstring autograft. Am J Sports Med 40:152-162

5. Coughlin MJ, Matt V, Schenck RC Jr (2002) Augmented lateral ankle reconstruction using a free gracilis graft. Orthopedics 25:31-35

6. Cross MJ, Roger G, Kujawa P, Anderson IF (1992) Regeneration of the semitendinosus and gracilis tendons following their transection for repair of the anterior cruciate ligament. Am J Sports Med 20:221-223

7. Culvenor AG, Perraton L, Guermazi A, Bryant AL, Whitehead TS, Morris HG et al (2016) Knee kinematics and kinetics are associated with early patellofemoral osteoarthritis following anterior cruciate ligament reconstruction. Osteoarthr Cartil 24:1548-1553

8. Eckstein F, Lemberger B, Gratzke C, Hudelmaier M, Glaser C, Englmeier $\mathrm{KH}$ et al (2005) In vivo cartilage deformation after different types of activity and its dependence on physical training status. Ann Rheum Dis 64:291-295

9. Engelken F, Wassilew Gl, Kohlitz T, Brockhaus S, Hamm B, Perka C et al (2014) Assessment of fatty degeneration of the gluteal muscles in patients with THA using MRI: reliability and accuracy of the Goutallier and quartile classification systems. J Arthroplast 29:149-153

10. Eriksson K, Hamberg P, Jansson E, Larsson H, Shalabi A, Wredmark T (2001) Semitendinosus muscle in anterior cruciate ligament surgery: morphology and function. Arthroscopy 17:808-817

11. Eriksson K, Kindblom LG, Hamberg P, Larsson H, Wredmark T (2001) The semitendinosus tendon regenerates after resection: a morphologic and MRI analysis in 6 patients after resection for anterior cruciate ligament reconstruction. Acta Orthop Scand 72:379-384

12. Farrokhi S, O'Connell M, Fitzgerald GK (2015) Altered gait biomechanics and increased knee-specific impairments in patients with coexisting tibiofemoral and patellofemoral osteoarthritis. Gait Posture 41:81-85

13. Ferretti A, Conteduca F, Morelli F, Masi V (2002) Regeneration of the semitendinosus tendon after its use in anterior cruciate ligament reconstruction: a histologic study of three cases. Am J Sports Med 30:204-207

14. Irie K, Tomatsu T (2002) Atrophy of semitzendinosus and gracilis and flexor mechanism function after hamstring tendon harvest for anterior cruciate ligament reconstruction. Orthopedics 25:491-495

15. Janssen RP, van der Velden MJ, Pasmans HL, Sala HA (2013) Regeneration of hamstring tendons after anterior cruciate ligament reconstruction. Knee Surg Sports Traumatol Arthrosc 21:898-905

16. Konrath JM, Vertullo CJ, Kennedy BA, Bush HS, Barrett RS, Lloyd DG (2016) Morphologic characteristics and strength of the hamstring muscles remain altered at 2 years after use of a hamstring tendon graft in anterior cruciate ligament reconstruction. Am J Sports Med 44:2589-2598

17. Lipscomb AB, Johnston RK, Snyder RB (1981) The technique of cruciate ligament reconstruction. Am J Sports Med 9:77-81

18. Louboutin H, Debarge R, Richou J, Selmi TA, Donell ST, Neyret P et al (2009) Osteoarthritis in patients with anterior cruciate ligament rupture: a review of risk factors. Knee 16:239-244

19. Maffulli N, Spiezia F, Testa V, Capasso G, Longo UG, Denaro V (2012) Free gracilis tendon graft for reconstruction of chronic tears of the Achilles tendon. J Bone Joint Surg Am 94:906-910

20. Marx RG, Stump TJ, Jones EC, Wickiewicz TL, Warren RF (2001) Development and evaluation of an activity rating scale for disorders of the knee. Am J Sports Med 29:213-218

21. Nakamae A, Deie M, Yasumoto M, Adachi N, Kobayashi K, Yasunaga Y et al (2005) Three-dimensional computed tomography imaging evidence of regeneration of the semitendinosus tendon harvested for anterior cruciate ligament reconstruction: a comparison with hamstring muscle strength. J Comput Assist Tomogr 29:241-245

22. Nakamura E, Mizuta H, Kadota M, Katahira K, Kudo S, Takagi K (2004) Threedimensional computed tomography evaluation of semitendinosus harvest after anterior cruciate ligament reconstruction. Arthroscopy 20:360-365

23. Ohkoshi Y, Inoue C, Yamane S, Hashimoto T, Ishida R (1998) Changes in muscle strength properties caused by harvesting of autogenous semitendinosus tendon for reconstruction of contralateral anterior cruciate ligament. Arthroscopy 14:580-584

24. Okahashi K, Sugimoto K, Iwai M, Oshima M, Samma M, Fujisawa $Y$ et al (2006) Regeneration of the hamstring tendons after harvesting for 
arthroscopic anterior cruciate ligament reconstruction: a histological study in 11 patients. Knee Surg Sports Traumatol Arthrosc 14:542-545

25. Papandrea P, Vulpiani MC, Ferretti A, Conteduca F (2000) Regeneration of the semitendinosus tendon harvested for anterior cruciate ligament reconstruction. Evaluation using ultrasonography. Am J Sports Med 28:556-561

26. Peterfy CG, Guermazi A, Zaim S, Tirman PF, Miaux Y, White D et al (2004) Whole-organ magnetic resonance imaging score (WORMS) of the knee in osteoarthritis. Osteoarthr Cartil 12:177-190

27. Petrie A (2006) Statistics in orthopaedic papers. J Bone Joint Surg Br 88: $1121-1136$

28. Rispoli DM, Sanders TG, Miller MD, Morrison WB (2001) Magnetic resonance imaging at different time periods following hamstring harvest for anterior cruciate ligament reconstruction. Arthroscopy 17:2-8

29. Scheibel M, Ifesanya A, Pauly S, Haas NP (2008) Arthroscopically assisted coracoclavicular ligament reconstruction for chronic acromioclavicular joint instability. Arch Orthop Trauma Surg 128:1327-1333

30. Schneider CA, Rasband WS, Eliceiri KW (2012) NIH image to ImageJ: 25 years of image analysis. Nat Methods 9:671-675

31. Simonian PT, Harrison SD, Cooley VJ, Escabedo EM, Deneka DA, Larson RV (1997) Assessment of morbidity of semitendinosus and gracilis tendon harvest for $\mathrm{ACL}$ reconstruction. Am J Knee Surg 10:54-59

32. Snow BJ, Wilcox JJ, Burks RT, Greis PE (2012) Evaluation of muscle size and fatty infiltration with MRI nine to eleven years following hamstring harvest for ACL reconstruction. J Bone Joint Surg Am 94:1274-1282

33. Tadokoro K, Matsui N, Yagi M, Kuroda R, Kurosaka M, Yoshiya S (2004) Evaluation of hamstring strength and tendon regrowth after harvesting for anterior cruciate ligament reconstruction. Am J Sports Med 32:1644-1650

34. Takeda Y, Kashiwaguchi S, Matsuura T, Higashida T, Minato A (2006) Hamstring muscle function after tendon harvest for anterior cruciate ligament reconstruction: evaluation with $\mathrm{T} 2$ relaxation time of magnetic resonance imaging. Am J Sports Med 34:281-288

35. Tashiro T, Kurosawa H, Kawakami A, Hikita A, Fukui N (2003) Influence of medial hamstring tendon harvest on knee flexor strength after anterior cruciate ligament reconstruction. A detailed evaluation with comparison of single- and double-tendon harvest. Am J Sports Med 31:522-529

36. Tashman S, Collon D, Anderson K, Kolowich P, Anderst W (2004) Abnormal rotational knee motion during running after anterior cruciate ligament reconstruction. Am J Sports Med 32:975-983

37. Tsifountoudis I, Bisbinas I, Kalaitzoglou I, Markopoulos G, Haritandi A, Dimitriadis A et al (2017) The natural history of donor hamstrings unit after anterior cruciate ligament reconstruction: a prospective MRI scan assessment. Knee Surg Sports Traumatol Arthrosc 25:1583-1590

38. Vourazeris JD, Lawless MW, Markert RJ, Stills HF, Boivin GP (2013) Semitendinosus muscle fatty infiltration following tendon harvest in rabbits. J Orthop Res 31:1234-1239

39. Williams GN, Snyder-Mackler L, Barrance PJ, Axe MJ, Buchanan TS (2004) Muscle and tendon morphology after reconstruction of the anterior cruciate ligament with autologous semitendinosus-gracilis graft. J Bone Joint Surg Am 86-A:1936-1946

\section{Publisher's Note}

Springer Nature remains neutral with regard to jurisdictional claims in published maps and institutional affiliations.

\section{Submit your manuscript to a SpringerOpen ${ }^{\circ}$ journal and benefit from:}

- Convenient online submission

- Rigorous peer review

- Open access: articles freely available online

- High visibility within the field

- Retaining the copyright to your article

Submit your next manuscript at $\boldsymbol{\nabla}$ springeropen.com 